\title{
Electrocoagulação com Cateter Venoso Periférico no Tratamento de Lagos Venosos
}

\author{
Miguel Pinto Gouveia'; Ana Isabel Gouveia²; Ana Brinca ${ }^{3}$; Ricardo Vieira ${ }^{4}$; Américo Figueiredo \\ 'Interno do Internato Complementar de Dermatologia e Venereologia/Resident, Dermatology and Venereology, Centro Hospitalar e \\ Universitário de Coimbra, Portugal \\ 2Interna do Internato Complementar de Dermatologia e Venereologia/Resident, Dermatology and Venereology, Centro Hospitalar \\ Lisboa Norte, Portugal \\ ${ }^{3}$ Assistente Hospitalar de Dermatologia e Venereologia, Unidade de Cirurgia Dermatológica/Consultant of Dermatology and \\ Venereology, Dermatologic Surgery Department, Centro Hospitalar e Universitário de Coimbra, Portugal \\ ${ }^{4}$ Professor Auxiliar em Dermatologia e Venereologia, Chefe da Unidade de Cirurgia Dermatológica/Assistant Professor of \\ Dermatology, Head of the Dermatologic Surgery Unit, Centro Hospitalar Universitário de Coimbra, Portugal \\ ${ }_{5}^{5}$ Professor Doutor em Dermatologia e Venereologia, Director do Serviço de Dermatologia e Venereologia do Centro Hospitalar e \\ Universitário de Coimbra/ Professor of Dermatology and Venereology and Head of Dermatology Department, Centro Hospitalar e \\ Universitário de Coimbra, Portugal
}

RESUMO - Lagos venosos são ectasias vasculares da derme superior localizados predominantemente nos lábios de indivíduos idosos. O seu tratamento pode ser desejável por motivos cosméticos ou funcionais, existindo uma miríade de métodos terapêuticos possíveis, incluindo ablação com laser, crioterapia ou cirurgia. A electrocoagulação é também um método eficaz, mas pode condicionar morbilidade do tecido são adjacente. Para minorar este dano, o recurso ao cateter venoso periférico para condução da energia até ao tecido alvo tem sido descrito como uma modificação vantajosa à técnica clássica.

Os autores descrevem o recurso à electrocoagulação com auxílio de cateter venoso periférico como alternativa acessível, simples e segura na abordagem dos lagos venosos.

PALAVRAS-CHAVE - Cateterismo Periférico; Dermatopatias Vasculares; Electrocoagulação; Electrocirurgia.

\section{Electrocoagulation with Intravenous Catheter in the Treatment of Venous Lakes}

ABSTRACT - Venous lakes comprise vascular ectasias of the upper dermis, usually seen in the lips of older patients. Treatment can be sought for cosmetic or functional reasons and therapeutic options include laser ablation, cryotherapy or surgery. Electrosurgery is a frequent and efficacious method in the resolution of venous lakes, although possibly damaging to the adjacent skin. In order to minimize skin damage, an intravenous catheter can be used to deliver electrocoagulation intralesionally from an electroscalpel, being described as an improvement of the classic procedure.

The authors describe this modified electrosurgical technique as a safe, simple and readily-accessible therapeutic option in the treatment of venous lakes.

KEY-WORDS - Catheterization, Peripheral; Electrocoagulation; Electrosurgery; Skin Diseases, Vascular.

Os lagos venosos correspondem a ectasias vasculares da derme superior, ocorrendo predominantemente nos lábios e pavilhões auriculares de indivíduos idosos. ${ }^{1}$ Apresentam-se como pápulas de tamanho variável (habitualmente entre 2 a
10 mm), de coloração azula-violácea, consistência mole e compressíveis. São tipicamente esporádicos, mas podem ser encontrados em associação a outras malformações vasculares ou constituir parte de síndromes (ex: síndrome de Sturge-Weber).
Correspondência: Dr. Miguel Pinto Gouveia

Serviço de Dermatologia do Centro Hospitalar e Universitário de Coimbra

Av. Bissaya Barreto, Praceta Prof. Mota Pinto - 3000-045 Coimbra, Portugal

Tel. +351239400420 - Fax: +351239400490

E-mail: miguelpgouveia@gmail.com
Recebido/Received

Outubro/October 2015

Aceite/Accepted

Novembro/November 2015 


\section{Caso Clínico}

Caracterizam-se histologicamente pela presença de uma vénula dilatada e congestionada na derme superficial. ${ }^{2}$

O tratamento pode ser equacionado por motivos cosméticos e funcionais, sobretudo pelo risco de hemorragia episódica que estas lesões que podem originar, optando-se frequentemente pela excisão cirúrgica ${ }^{3}$ ou crioterapia ${ }^{4}$ dada a maior familiaridade com estas técnicas. No entanto existem múltiplos procedimentos terapêuticos que, apesar de serem genericamente eficazes, divergem no preço, acessibilidade e efeitos adversos. Os métodos descritos incluem a escleroterapia percutânea (ex: polidocanol 1\%), ${ }^{5}$ coagulação por radiação infravermelha, ${ }^{6} \mathrm{IPL},{ }^{7}$ ablação com múltiplos tipos de laser (árgon, ${ }^{8}$ $\mathrm{PDL},{ }^{9}$ díodo, ${ }^{10} \mathrm{CO}_{2}{ }^{11}{ }^{11} \mathrm{Nd}: \mathrm{YAG}^{12}$ e combinação PDL/Nd:YAG ${ }^{13}$ ) além de diversas modalidades de electrocoagulação. ${ }^{14,15}$

Recordamos uma técnica simples, minimamente invasiva, eficaz e barata para resolução dos lagos venosos de dimensão pequena a moderada. Esta técnica consiste na electrocoagulação da lesão, usando um cateter venoso periférico para conduzir a energia eléctrica ao tecido alvo. ${ }^{15} \mathrm{Na}$ preparação do cateter, é criada uma janela na porção proximal da cânula de plástico, expondo a agulha metálica e permitindo o contacto com o bisturi elétrico monopolar (Fig. 1). Consegue-se, assim, a passagem da energia para a ponta do cateter, de forma a coagular o lago venoso com dano mínimo para os tecidos adjacentes, em virtude do isolamento que a cânula de plástico oferece.

Durante o procedimento observa-se a contracção da lesão e a alteração da habitual cor azul para cinzento-esbranquiçado. No caso de lagos venosos de maior dimensão, o cateter pode ser mobilizado de modo a coagular sequencialmente toda a lesão (Fig. 2). A eventual hemorragia resultante da remoção do cateter é discreta e poderá ser controlada com compressão. Após o procedimento sugere-se aplicação regular de solução antissética adequada até à cicatrização total. Consegue-se habitualmente resolução completa numa única sessão terapêutica (Fig. 3) e os resultados cosméticos são bons,

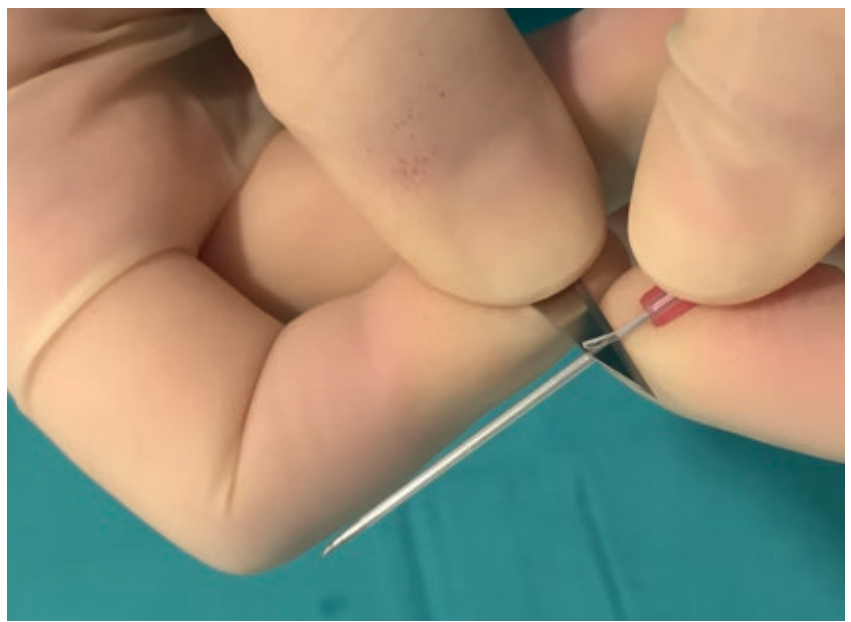

Figura 1 - Preparação do cateter venoso periférico, recorrendo a uma lâmina de bisturi para a criação de uma janela na porção proximal da cânula de plástico, expondo a agulha metálica.

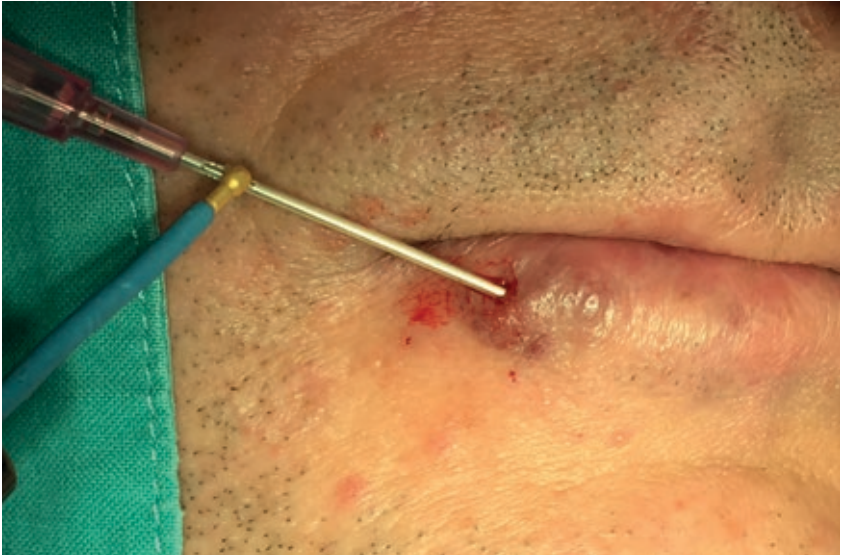

Figura 2 - Mobilização intra-lesional da ponta do cateter venoso central, com aplicação de energia do bisturi eléctrico monopolar através da janela previamente realizada na cânula de plástico.

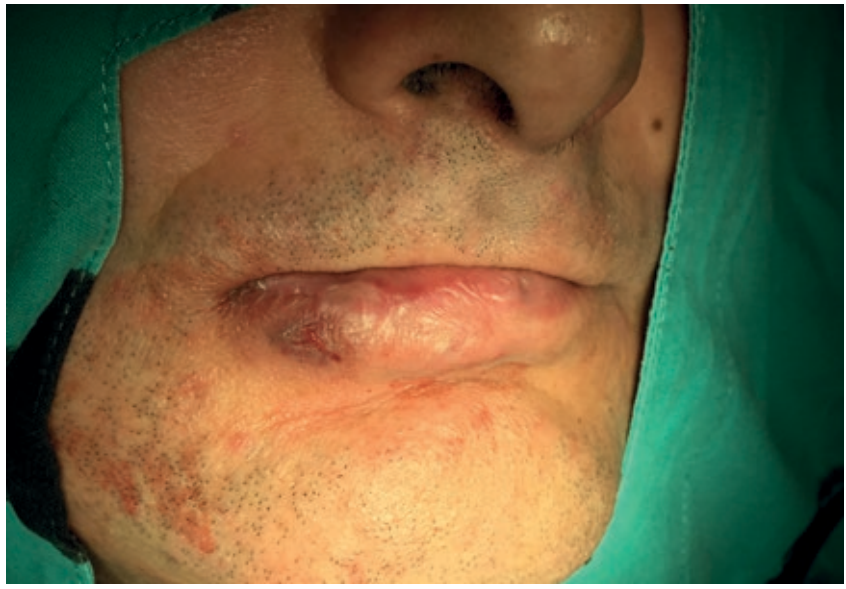

Figura 3 - Resultado intra-operatório imediato, com transformação para coloração acinzentada da lesão.

sendo um procedimento muito bem tolerado, decorrendo sob anestesia local.

Pela simplicidade, eficácia, rapidez e baixo custo, sugerimos este método como primeira opção no tratamento de lagos venosos.

Conflitos de interesse: Os autores declaram não possuir conflitos de interesse. Suporte financeiro: $O$ presente trabalho não foi suportado por nenhum subsídio ou bolsa. Direito à privacidade e consentimento escrito: Os autores declaram que pediram consentimento ao doente para usar as imagens no artigo.

Conflicts of interest: The authors have no conflicts of interest to declare. Financing Support: This work has not received any contribution, grant or scholarship. Privacy policy and informed consent: The authors declare that the patient gave written informed consent for the use of its photos in this article. 


\section{Caso Clínico}

\section{REFERÊNCIAS}

1. Bean WB, Walsh JR. Venous lakes. AMA archives of dermatology. 1956; 74:459-63.

2. Mckee P, Calonje E, Granter S. Pathology of the skin, with clinical correlations. 3rd ed. Philadelphia: Mosby; 2005.

3. Huang CC, Arpey CJ. The lips. Excision and repair. Dermatologic clinics. 1998; 16(1):127-43.

4. Suhonen R, Kuflik EG. Venous lakes treated by liquid nitrogen cryosurgery. Br J Dermatol. 1997; 137:1018-9.

5. Kuo HW, Yang $\mathrm{CH}$. Venous lake of the lip treated with a sclerosing agent: report of two cases. Dermatol Surg. 2003; 29:425-8.

6. Ah-Weng A, Natarajan S, Velangi S, Langtry JA. Venous lakes of the vermillion lip treated by infrared coagulation. Br J Oral Maxillofac Surg. 2004; 42:251-3.

7. Jay $\mathrm{H}$, Borek $\mathrm{C}$. Treatment of a venous-lake angioma with intense pulsed light. Lancet. 1998; 351:112.

8. Neumann RA, Knobler RM. Venous lakes (Bean-Walsh) of the lips--treatment experience with the argon laser and 18 months follow-up. Clin Exp Dermatol. 1990; 15:115-8.

9. Cheung ST, Lanigan SW. Evaluation of the treatment of venous lakes with the 595-nm pulsed-dye laser: a case series. Clinical Exp Dermatol. 2007; 32:148-50.

10. Azevedo LH, Galletta VC, Eduardo Cde P, Migliari DA. Venous lake of the lips treated using photocoagulation with high-intensity diode laser. Photomed Laser Surg. 2010; 28:263-5.

11. del Pozo J, Pena C, Garcia Silva J, Goday JJ, Fonseca E. Venous lakes: a report of 32 cases treated by carbon dioxide laser vaporization. Dermatol Surg. 2003; 29:308-10.

12. Bekhor PS. Long-pulsed Nd:YAG laser treatment of venous lakes: report of a series of 34 cases. Dermatol Surg. 2006; 32:1151-4.

13. 13. Roncero M, Canueto J, Blanco S, Unamuno P, Boixeda P. Multiwavelength laser treatment of venous lakes. Dermatol Surg. 2009; 35:1942-6.

14. 14. Weiss J, Weiss KD, Ross AL, Weiss E. A simplified minimally invasive technique for the treatment of venous lakes. Dermatol Online J. 2014; 20:21257.

15. 15. Guarneri GF, De Biasio F, Semprini G, Cracco S, Parodi PC. A simple method to treat venous lakes. Dermatol Surg. 2010; 36:1586-7. 